\title{
Recurrent genital ulceration: a case of dermatitis herpetiformis
}

\author{
K Sivakumar, D C Coelho, R Basu Roy
}

Recurrent genital ulceration is commonly seen in genitourinary medicine clinics and the majority of these ulcers are due to infections. We report a case of a 28 year old man suffering from recurrent genital ulcers due to dermatitis herpetiformis.

\section{Case history}

A 38 year old married Caucasian presented to our department with a two week history of an itchy vesicular penile eruption. At the onset there had been itchiness and erythema followed by oedema and blistering of the prepuce, developing over a 24-hour period. The blisters ruptured leaving superficial ulcers (fig 1). A clinical diagnosis of herpes was made and swabs from the lesions were sent for herpes simplex virus (HSV) culture. The HSV culture was negative and his initial symptoms improved without any specific treatment, within two weeks.

His genital signs and symptoms recurred three times during the next twelve months, before he developed similar lesions over his right shoulder, elbows and sacrum. At this stage the possibility of vesiculo-bullous skin lesions including dermatitis herpetiformis was considered and further investigations were undertaken.

\section{INVESTIGATION AND MANAGEMENT}

Repeated HSV cultures and tests for other STDs were negative. A full blood count, erythocyte sedimentation rate, urea, electrolytes, LFTs, calcium and serum proteins were within the normal range. Histology of the perilesional skin biopsy showed the edge of a supepidermal bulla and microabscesses at the tips of the dermal papillae (fig 2). These changes are suggestive of dermatitis herpetiformis. A duodenal biopsy showed severe villous atrophy, a dense lymphocytic and plasmacytic infiltrate in the lamina propria, and lymphocytes in the surface epithelium, all changes suggestive of a gluten-sensitive enteropathy. The immunofluores-

Department of Genitourinary Medicine, Royal Victoria Hospital, Gloucester Road, Bournemouth BH7 6JF, UK

K Sivakumar, D C Coelho, R Basu Roy cence test for IgA deposits, unfortunately done only once, was negative, possibly a technical false negative.

On clinical and histological grounds a diagnosis of dermatitis herpetiformis was made. The patient was started on dapsone $150 \mathrm{mg}$ daily. With dapsone his symptoms improved dramatically within $\mathbf{4 8}$ hours. To date he continues to remain well and symptomfree on a smaller dose of dapsone.

\section{Discussion}

The number of new cases of herpes simplex infections reported in the United Kingdom (from clinic returns to health departments) increased from 7,722 in 1977 to 16,699 in $1987^{1}$ and it is the commonest cause for genital ulceration in the United Kingdom at present. The diagnosis is often made clinically and confirmed by positive HSV culture. The ability to isolate virus from the lesions varies over the course of the disease. ${ }^{2} \mathrm{HSV}$ can be isolated from over $90 \%$ of vesicular or pustular lesions but the isolation of virus

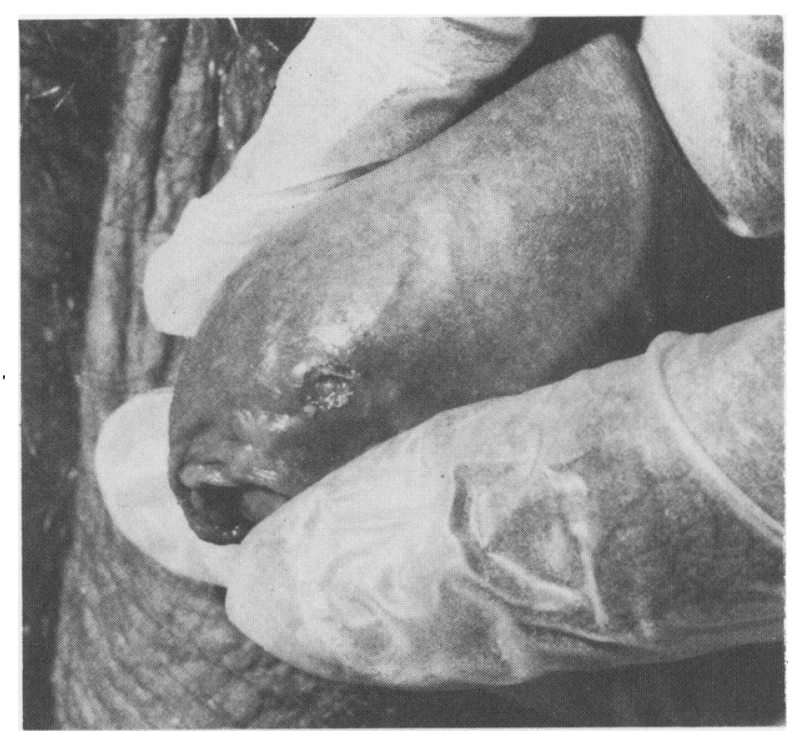

Figure 1 Superficial ulcers of prepuce 


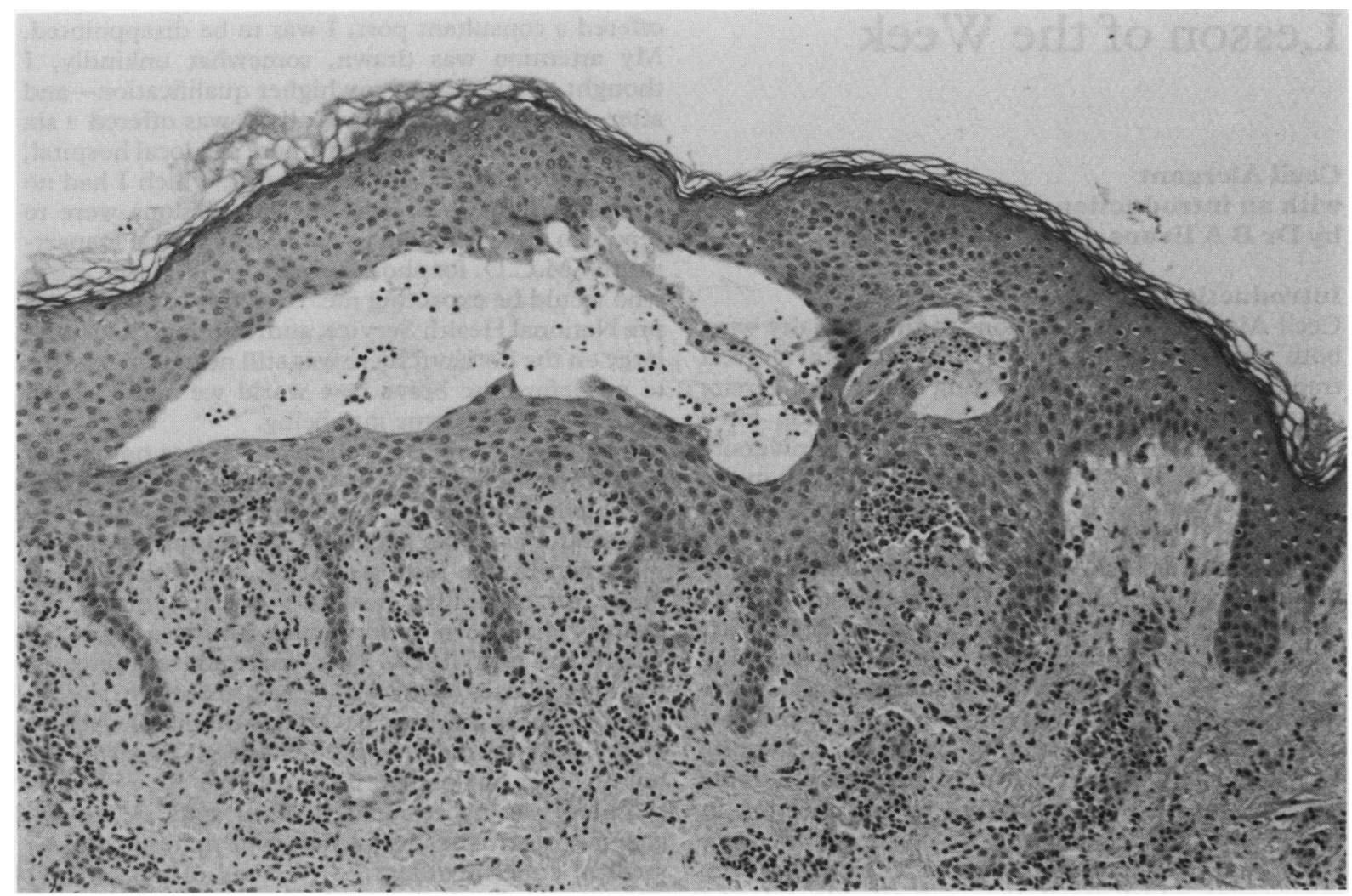

Figure 2 Edge of a bulla and microabscesses at the tips of dermal papillae. (Haematoxylin and eosin, $\times 40$ )

is markedly reduced in the ulcerative and crust stages of disease. Therefore the lack of isolation of $\mathrm{HSV}$ does not rule out HSV infection. When patients present with recurrent genital ulceration, with several negative HSV cultures and tests for other STDs, other rare noninfectious causes of genital ulceration, such as inflammatory bowel disease, Behcet's syndrome and vesiculo-bullous skin diseases should be considered in the differential diagnosis. This would help exclude the wrong labelling of patients with genital ulcers as herpes genitalis. Herpes is still a highly emotive condition and a wrong diagnosis is to be avoided as far as is practicable.
We thank Consultants: C Loehry for the duodenal biopsy, P Goodwin for treatment and M Lesna for histology and photomicrophy.

1 New cases of sexually transmitted disease reported by NHS GUM clinics England 1977-1987 (table 1). From SBH601987 D H Statistics and Research (SR2B)-March 1989.

2 Mosley RC, Corey L, Benjamin D, Winter C, Remington ML: Comparison of viral isolation, direct immunofluorescence and indirect immunoperoxidase techniques for detection of genital herpes simplex virus infection. J Clin Microbiol 1981;13: 